\title{
STUDY OF TURBULENT HYPERSONIC FLOW PAST ELONGATED BLUNT-NOSED CONES
}

\author{
I. I. Palamarchuk, G. A. Tirskii, S. V. Utyuzhnikov, \\ and M. O. Fridlender
}

UDC 533.6.011.55:532.517.4

Axisymmetric turbulent hypersonic perfect-gas flow past slightly blunted cones is considered. The influence of the absorption of the entropy layer, developing on an elongated body, on friction and heat transfer to the body surface is studied using the viscous shock layer model. Correlations are established for the heat flux and the friction coefficient along the cone surface at large distances from the forward stagnation point. The results are presented together with their correlations in the framework of the viscous shock layer model and an approximate model of the boundary layer type, taking into account the entropy layer absorption. A generalization of the Reynolds analogy is obtained.

There are many papers dealing with the calculation of the friction and heat transfer in flow past blunt bodies within the framework of the classical theory of the turbulent boundary layer (see [1]). The absorption of the entropy layer by the boundary layer may be regarded as the principal second-order effect in boundary layer theory. Approximate methods have been developed to take this effect into account (see [2-6]). It should be noted that the entropy layer is absorbed more rapidly by a turbulent than by a laminar boundary layer, due to the greater boundary layer thickness; in this case the absorption length is 5-50 bluntness radii. In [2-4] the effect of the absorption of the entropy layer by the boundary layer was taken into account by means of additional calculations for the flow parameters at the outer edge of the boundary layer, using the mass flow rate global balance method. The authors of [5] and [6] have proposed a technique based on the mass-average method. All these approaches enable the entropy layer absorption to be taken into account without solving a set of equations more intricate than that of boundary layer theory. All these approaches need theoretical or experimental verification.

The similitude criteria for turbulent boundary layers on blunt-nosed cones were analyzed in $[4,6]$. In [4] the similitude parameters determining the heat transfer to the cone surface were established for the entropy layer absorption regime over the following ranges of the governing parameters: cone half-angle $\theta_{k}=10 \mathrm{deg}$, Reynolds number $\operatorname{Re}_{\infty}=1.0 \cdot 10^{5}-3.1 \cdot 10^{5}, \mathrm{Mach}$ number $M_{\infty}=6-8$, temperature factor $t_{w}=0.40-0.52$ (here, $t_{w}=T_{w} / T_{0}, T_{w}$ being the wall temperature and $T_{0}-$ the stagnation temperature). The similitude criteria governing hypersonic flow past slender blunt bodies in the turbulent boundary layer regime were determined in [6].

Our aim is to study numerically the problem of hypersonic turbulent flow past blunt-nosed cones using the viscous shock layer model; to estimate the errors of the various approximate theories; and to establish the similitude criteria for the heat transfer at large distances from the bluntness and for the friction at both large and intermediate distances.

\section{PROBLEM FORMULATION AND METHOD OF SOLUTION}

The set of viscous shock layer equations, described in [7,8], is used as the gasdynamic flow model. All the terms of the Euler equations are present in the equations of this set, together with all the second-order terms of asymptotic boundary layer theory. The two-layer Cebeci-Smith model [1] is used to describe the flow turbulence. The gas flow between the bow shock and the body surface is governed by the viscous shock layer equations for a perfect gas $[7,8]$ :

$$
\begin{gathered}
\frac{\partial}{\partial x}(r p u)+\frac{\partial}{\partial y}\left(H_{1} r p v\right)=0, \quad P\left(\frac{u}{H_{1}} \frac{\partial u}{\partial x}+v \frac{\partial u}{\partial y}+\frac{u v}{R(x) H_{1}}\right)=-\frac{1}{H_{1}} \frac{\partial p}{\partial x} \\
+\frac{1}{\operatorname{Re}_{\infty} H_{1}^{2} r} \frac{\partial}{\partial y}\left[r H_{1}^{2} \mu\left(\frac{\partial u}{\partial y}-\frac{u}{R(x) H_{1}}\right)\right], \quad P\left(\frac{u}{H_{1}} \frac{\partial v}{\partial x}+v \frac{\partial v}{\partial y}-\frac{u^{2}}{R(x) H_{1}}\right)=-\frac{\partial p}{\partial y} \\
P\left(\frac{u}{H_{1}} \frac{\partial H}{\partial x}+v \frac{\partial H}{\partial y}\right)=\frac{1}{\operatorname{Re}_{\infty} H_{1} r} \frac{\partial}{\partial y}\left\{r H_{1} \frac{\mu}{\operatorname{Pr}}\left[\frac{\partial H}{\partial y}+\frac{V_{\infty}^{2}}{H_{\infty}}\left(\frac{\operatorname{Pr}-1}{2} \frac{\partial\left(u^{2}\right)}{\partial y}-\frac{\operatorname{Pr}}{R(x) H_{1}} u^{2}\right)\right]\right\}
\end{gathered}
$$

Moscow. Translated from Izvestiya Rossiiskoi Akademii Nauk, Mekhanika Zhidkosti i Gaza, No.6, pp. 123-128, November-December, 1993. Original article submitted July 22, 1992. 


$$
H=\left(\frac{x}{x-1} \frac{p}{p}+\frac{u^{2}+v^{2}}{2}\right) \frac{V_{\infty}^{2}}{H_{\infty}}, \quad \mu=\mu_{t}+\mu_{t}, \quad \lambda=\lambda_{t}+\lambda_{t}
$$

The set of equations (1.1) is written down in the curvilinear orthogonal reference frame $(x, y)$ fitted to the body. All the variables have been nondimensionalized; $p-k \rho_{\infty} V_{\infty}^{2}$; the linear ones have been divided by the bluntness radius, and all the rest by their values in the free stream. Here, $u$ and $v$ are the nondimensional velocity components in the $x$ - and $y$-directions; $p, \rho$, and $H$ are pressure, density, and total enthalpy, respectively; $\mu$ is the effective viscosity coefficient, $\mu_{l}$ is the Sutherland laminar viscosity coefficient, and $\mu_{t}$ is the turbulent viscosity coefficient, which is determined according to the model proposed in [1]; $\lambda, \lambda_{l}$, and $\lambda_{t}$ are the effective, laminar, and turbulent thermal conductivity coefficients, respectively; $\operatorname{Pr}=\mu c_{p} / \lambda$ is the effective Prandtl number; $R_{w}$ is the bluntness radius, $H_{1}=1+y / R(x)$ is the scale factor, $R(x)$ being the body curvature radius and $r$ the distance of any point from the axis of symmetry; $x=c_{p} / c_{v}$ is the specific heat ratio. A subscript " $\infty$ " is used to denote free-stream conditions.

The bow shock is assumed to be thin. The impermeability and no-slip conditions are imposed on the body surface, together with that of constant wall temperature. The generalized Rankine-Hugoniot relations are assumed to be satisfied at the shock wave:

$$
\begin{gathered}
K_{s}=\frac{P_{\infty}}{\rho_{s}}=\frac{x-1}{2}\left(\frac{1}{\left(\mathrm{M}_{\infty}\right)^{2}(\sin \beta)^{2}}+\frac{2}{\chi+1}\right) \\
u_{s}=\cos \beta \cos \beta_{s}+K_{s} \sin \beta \sin \beta_{s}-\frac{\mu_{s}}{\sin \beta} \frac{1}{\operatorname{Re}}\left(\frac{\partial u}{\partial y}-\frac{u}{R(x) H_{1}}\right)_{s}, \quad p_{s}=\left(1-K_{s}\right)(\sin \beta)^{2}+\frac{1}{\chi\left(\mathrm{M}_{\infty}\right)^{2}} \\
v_{s}=u \operatorname{tg} \beta_{s}-K_{s} \frac{\sin \beta}{\cos \beta_{s}}, \quad H_{s}=1-\frac{1}{\operatorname{Re}{ }_{\infty}} \frac{\mu_{s}}{\operatorname{Pr} \sin \beta}\left(\frac{\partial H}{\partial y}+\frac{\operatorname{Pr}-1}{2} \frac{V_{\infty}^{2}}{H_{\infty}} \frac{\partial\left(u^{2}\right)}{\partial y}-\frac{V_{\infty}^{2}}{H_{\infty}} \frac{\operatorname{Pr}}{\mathrm{R}(x) \mathrm{H}_{1}} u^{2}\right)_{s}
\end{gathered}
$$

Here, the subscript " $s$ " denotes values behind the bow shock, $\beta_{s}$ is the angle between the tangent to the shock and axis of symmetry, $\beta$ is the angle between the tangents to the shock and to the body for a given $x$.

The viscous shock layer equations were integrated by a numerical method based on global iterations. This approach, very economical as regards computer resources, enables the computer time to be reduced by a factor of approximately 10 , as compared with time-dependent methods. For calculating the flow past elongated bodies the whole flow region was split into blocks, and a step-by-step approach was used to integrate the flow equation in each block [8]. The difference scheme was of second order of approximation in the derivatives with respect to $x$ and of fourth order in those with respect to $y$. The grid points were distributed nonuniformly in the normal direction, the magnitude of the mesh step at each grid point depending on the behavior of the required functions in the vicinity of that point. The distribution of the grid points in the $x$-direction was chosen so as to make one of these points coincide with the junction of the spherical nose and the conical afterbody, where the body contour has a curvature discontinuity. In order to calculate the flow parameters at the point of discontinuity with second order of approximation the exact relations for the discontinuities in the first and second derivatives of the required functions were used.

\section{DISCUSSION OF RESULTS}

The hypersonic turbulent flow past cones with half-angles $\theta_{k}=10,20$, and $30^{\circ}$ and surface temperatures $t_{w}=0.05,0.2$, 0.6 was calculated for $\operatorname{Re}_{\infty}=10^{6}, 10^{7}, 10^{8}$ and $\mathrm{M}_{\infty}=6,8,10$. The flow equations were integrated from the forward stagnation point over a distance $x=200 R_{w}$.

Figure 1 shows the calculated plots of the Stanton number St (curve 1) and the friction coefficient $C_{f}$ (curve 2) against the distance $s$ measured in bluntness radii from the stagnation point along the cone surface:

$$
\mathrm{St}=q /\left[\rho_{\infty} V_{\infty} c_{p} T_{0}\left(1-t_{w}\right)\right], \quad C_{f}=2 \tau /\left(\rho_{\infty} V_{\infty}^{2}\right), \quad s=x / R_{w}
$$

where $q=\lambda \partial T / \partial y, \tau=\mu \partial u / \partial y$ (here all the variables are dimensional).

Figure 1 shows that the Stanton number reaches a maximum not exactly at the stagnation point, as in laminar flow, but rather in its vicinity. Then St diminishes due to the increase in flow velocity and rises again because of the absorption of the entropy layer by the boundary layer. The subsequent decrease in St is due to the growth in boundary layer thickness. The location of the second maximum depends on the cone half-angle and the Reynolds number, while its magnitude also depends on $\mathbf{M}_{\infty}$ and $t_{w}$.

The plot of $C_{f}$ against the distance along the body contour is also characterized by two maxima. The first of these is located 


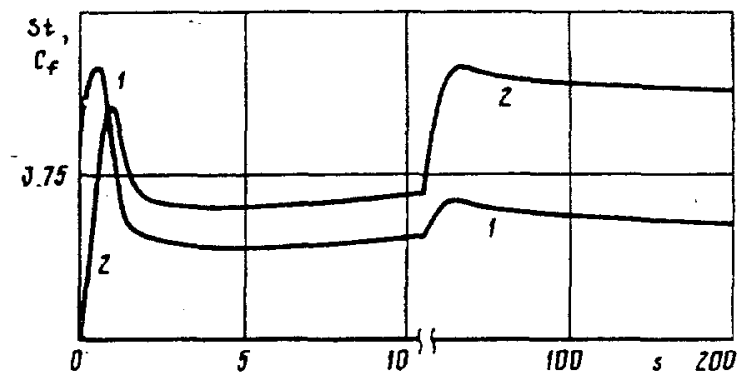

Fig 1

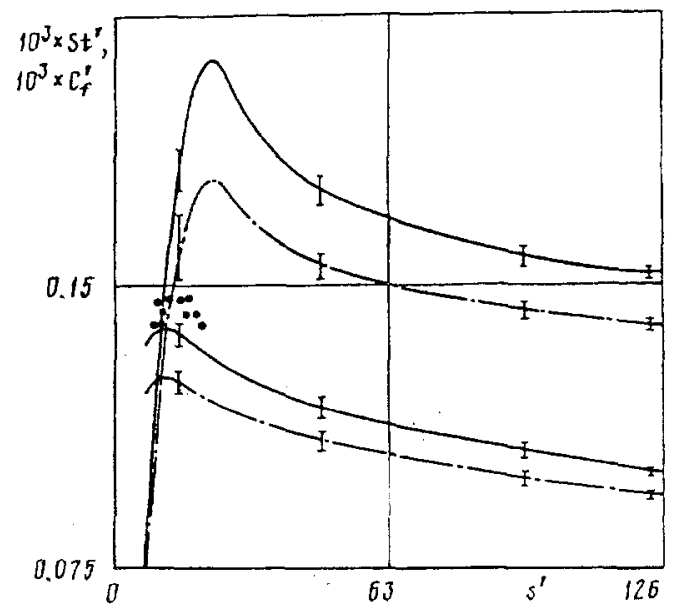

Fig 2

within the nose region, while the second is situated in the region where the entropy layer is being swallowed up by the boundary layer $(15 \leq s \leq 60)$.

The location and the magnitude of the second maximum depend on the same factors as in the case of the Stanton number.

The results of the calculations made in [4] were presented in that paper as $\mathrm{St}^{*}\left(s^{*}\right)$, where $\mathrm{St}^{*}$ is the modified Stanton number and $s^{*}$ is the modified distance from the stagnation point, measured in fractions of the distance between the stagnation point and the entropy layer absorption point. In this modification the Reynolds number and the cone half-angle appear explicitly, while the Mach number and the temperature factor enter implicitly, by way of the velocity and density values at the outer edge of the boundary layer.

Attempts to use the dependent and independent similitude criteria at distances greater than the absorption length and over a wider range of flow parameters have proved fruitless. The introduction of an explicit dependence of the boundary layer thickness on $t_{w}$ and $\mathrm{M}_{\infty}$ has likewise not led to the desired results. We therefore obtained correlations for the Stanton number and the friction coefficient derived empirically in processing our results and those of [2]:

$$
\begin{gathered}
\mathrm{St}^{\prime}=\mathrm{St}^{\prime}\left(s^{\prime}\right), \quad C_{f}^{\prime}=C_{f^{\prime}}\left(s^{\prime}\right), \quad \mathrm{St}^{\prime}=\mathrm{St}\left[\left(1.1-t_{w}\right)^{-1}\left(1-\frac{6}{\mathrm{M}_{\infty}^{2}}\right)^{-1}\left(\lg _{\infty} \mathrm{Re}_{\infty}\right)^{3}\left(2 \sin \theta_{k}\right)^{\alpha_{k}}\right] \\
C_{f}^{\prime}=C_{f}\left[\left(2.1-t_{w}\right)^{-1}\left(1-\frac{7}{\mathrm{M}_{\infty}^{2}}\right)^{-1}\left(\lg \operatorname{Re}_{\infty}\right)^{2,1}\left(2 \sin \theta_{k}\right)^{\beta k}\right] \\
s^{\prime}=\frac{x}{R_{w}}\left(2 \sin \theta_{k}\right)^{2} \exp \left(10^{-8} \operatorname{Re}_{\infty}\right), \quad \alpha_{k}=-1.27+0.2\left(2 \sin \theta_{k}\right)^{2}, \quad \beta_{k}=-1+1.5\left(2 \sin \theta_{k}\right)^{\downarrow}
\end{gathered}
$$

Figure 2 shows the $\mathrm{St}^{\prime}\left(s^{\prime}\right)$ plots over the range $s^{\prime}=15-125$ and the $C_{f}^{\prime}\left(s^{\prime}\right)$ plots for $s^{\prime}=2-125$. The continuous curve relates to the viscous shock layer model, and the chain curve to the boundary layer model, with account for entropy layer absorption [2]. The spread of the numerical data is represented by vertical bars.

The following approximate formulas are proposed for $\mathrm{St}^{\prime}\left(s^{\prime}\right)$ and $C_{f}^{\prime}\left(s^{\prime}\right)$ within the framework of the viscous shock layer model:

$$
\mathrm{St}^{\prime}=0.197\left(s^{\prime}\right)^{-0.139}, \quad C_{f}^{\prime}=C_{f 1}(1-a)+C_{f 2} a
$$

where

$$
C_{f_{1}}=0.0500+0.0130 s^{\prime}, \quad C_{f_{2}}=0.2985\left(s^{\prime}\right)^{-0.139}, \quad a=\exp \left[-\left(9 / s^{\prime}\right)^{3}\right]
$$

The points in Fig. 2 represent the experimental results of [3]. The experiments were carried out on a blunt-nosed cone with $\theta_{k}=10^{\circ}$ at $t_{w}=0.4, \mathrm{M}_{\infty}=8$, and $\operatorname{Re}_{\infty}=10^{5}$.

As $\operatorname{Re}_{\infty}$ increases from $10^{6}$ to $10^{8}$, the maxima of the $C_{f}$ and St distributions shift downstream, diminishing in magnitude. The situation is the same when the cone half-angle $\theta_{k}$ is reduced from 30 to $10^{\circ}$. A change in $\mathbf{M}_{\infty}$ and $t_{w}$ affects the magnitude of the second maximum but has no significant effect on its location. Thus, an increase in Mach number from 6 to 10 leads to a rise in the maximum values of St and $C_{f}$, while an increase in $t_{w}$ from 0.05 to 0.6 leads to a fall.

The spread of the correlated numerical data (see Fig. 2) does not exceed $3 \%$ in the far zone and $10 \%$ in the region of the 


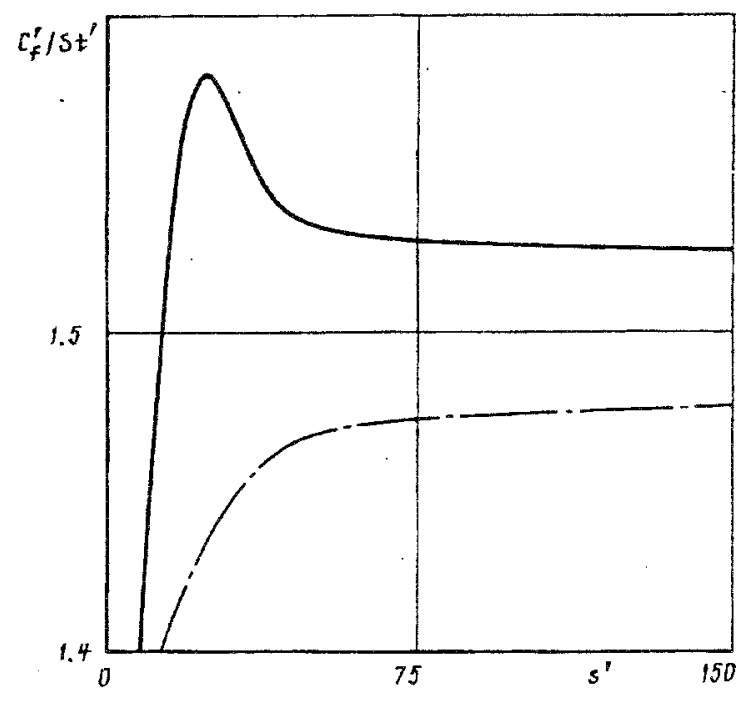

Fig 3

second maximum for the heat flux and $4 \%$ and $14 \%$, respectively, for the friction coefficient. The experimental data lie within this range, thus confirming our conclusions.

The results presented demonstrate that the magnitudes of the Stanton number calculated according to the viscous shock layer model exceed by 5-8\% those calculated by the approximate method of [2]; the corresponding figures for $C_{f}$ are $2-20 \%$.

Figure 3 shows the generalization of the Reynolds analogy illustrating the relations (2.1) for $\mathrm{St}^{\prime}$ and $C_{f}^{\prime}$. The ratio of nondimensional heat flux to nondimensional friction not only depends on the parameters $t_{w}, \mathbf{M}_{\infty}, \operatorname{Re}_{\infty}$, and $\theta_{k}$ but also varies along the body contour. At $s^{\prime}>50$ the ratio $C_{f}^{\prime} / \mathrm{St}^{\prime}$ is constant, does not depend on the method of calculation and is equal to $3 / 2$, with a probable error of approximately $1 \%$. The calculated results show that at distances $10<s^{\prime}<50$ from the stagnation point the dependence of the heat flux-to-friction ratio is nonmonatonic.

In conclusion, we note that the calculation of fully turbulent flow past elongated bodies starting from the stagnation point is permissible, since the heat flux values in the region where the laminar-turbulent transition is complete coincide with those corresponding to earlier transition $[3,9,10]$.

\section{REFERENCES}

1. T. Cebeci and A. M. O. Smith, Analysis of Turbulent Boundary Layers, No. 4, Academic Press, New York (1974), p. 92.

2. N. P. Kolina, A. I. Pyatnova, and E. E. Solodkin, "Influence of entropy layer absorption on the aerodynamical coefficients of elongated bodies in various boundary layer flow regimes," Tr. TsAGI, No. 2107, 121 (1981).

3. N. P. Kolina, Yu. Yu. Kolochinskii, and A. Ya. Yushin, "Influence of entropy layer absorption on the heat transfer to the surface of a cone in a supersonic stream," Uch. Zap. TSAGI, 16, No. 3, 21 (1985).

4. Yu. N. Ermak, N. P. Kolina, and A. Ya. Yushin, "Heat transfer on the lateral surface of a blunt cone in the case of absorption of an entropy layer by a laminar and turbulent boundary layer," Zh. Prikl. Mekh. Tekh. Fiz., No. 5, 65 (1985).

5. B. A. Zemlyanskii and G. N. Stepanov, "Calculation of heat transfer accompanying hypersonic three-dimensional airflow over slender blunt-nosed bodies," Izv. Akad. Nauk SSSR, Mekh. Zhidk. Gaza, No. 5, 173 (1981).

6. V. V. Lunev, "On the similarity criteria for hypersonic turbulent boundary layer flows over slender bodies," Izv. Akad. Nauk SSSR, Otd. Tekh. Nauk. Mekh. Mashinostr., No. 4, 13 (1962).

7. S. A. Vasil'evskii, G. A. Tirskii, and S. V. Utyuzhnikov, "Numerical method of solving the viscous shock layer equations," $Z$ h. Vychisl. Mat. Mat. Fiz., 27, 741 (1987).

8. S. V. Utyuzhnikov, "Numerical solution of the complete set of viscous shock layer equations as applied to the problem of hypersonic flow past a blunt-nosed body," Chislennye Metody Mekh. Splosh. Sredy, 17, No. 6, 125 (1986).

9. L. E. Ericsson, "Effect of nose bluntness and cone angle on slender vehicle transition," AIAA J., 26, 1168 (1988).

10. E. V. Zoby and R. A. Thompson, "Flowfield and vehicle parameter influence on hypersonic heat transfer and drag," $J$. Spacecr., 27, $361(1990)$. 